\title{
Collaborative learning through practices of group cognition
}

\author{
Gerry Stahl, Drexel University, Philadelphia, USA, Gerry@GerryStahl.net
}

\begin{abstract}
While there is evidence that collaborative learning consists largely of group-level practices, there has been little analysis and description of these processes as such; learning has generally been studied at the individual unit of analysis. Our research, in contrast, focuses on describing the interactional small-group practices that take place in learning contexts. This paper considers these practices and how they work together to form the foundation for effective collaborative learning activities. It analyzes collaborative learning activities in a paradigmatic CSCL setting to discuss such small-group practices as: resolving cognitive conflict, pursuing inquiry, maintaining a group problem space and coordinating multiple modes of reasoning. These have broad implications for foundational issues of temporality, indexicality and group cognition.
\end{abstract}

\section{Individual and Group Learning}

Learning has traditionally been considered a change in the knowledge of individual minds. More recently, it has been conceptualized at the opposite extreme in terms of participation in communities of practice (Lave \& Wenger, 1991). In general, learning is conceptualized at the individual level and group knowledge building at the community level, despite evidence of the centrality of the small group, particularly in CSCL settings. An intermediate position between these two extremes is to consider how learning takes place in the practices of small groups (Stahl, 2006a). This is particularly appropriate for CSCL contexts, which are designed to support the building of knowledge in small groups and where learning is promoted through the effective interaction of students in online small groups.

Recent work in CSCL and the learning sciences indicates that learning takes place differently in small groups than when students are working on their own (e.g., Barron, 2003; Cohen et al., 2002; Schwartz, 1995). That is, if one measures individual learning as a difference between knowledge before and after some intervention, the inclusion of group work as part of the intervention makes a difference. These studies speculate that the difference is due to group processes, such as the practices involved in making ideas or concepts explicit and explaining them to group members. However, these studies were not specifically designed to capture the group processes and to describe how they were involved in group learning. Unfortunately, studies of learning rarely focus on the small-group processes themselves as activities of knowledge building.

We have conducted a research project during the past five years to explore the group processes involved in learning activities in a paradigmatic CSCL environment: the Virtual Math Teams (VMT) Project at Drexel University. It is based on our theory of group cognition (Stahl, 2006a), which we are still elaborating as a basis for understanding core processes underlying CSCL theory, design and practice. Investigations in VMT are designed to explore group practices in online collaborative learning of mathematics. The supporting technology is instrumented to capture all the data needed to observe group phenomena rigorously. We focus our analysis on case studies - some quite brief, others extending across several chat sessions.

Our publications to date have presented focused aspects of this research or described specific practices that seem to be important for understanding collaborative learning. In more theoretical reflections on this, my contribution to the CSCL2 book argued for "rediscovering the CSCL" that tends to be lost in research at the individual-student or isolated-utterance unit of analysis (Stahl, 2006a, Ch 10). At the CSCL 2002 conference, I proposed using interaction analysis to study group perspectives and collaborative knowledge building (Stahl, 2006a, Ch 11). My CSCL 2003 paper differentiated individual interpretation processes from the group meaningmaking practices (Stahl, 2006a, Ch 16). For CSCL 2005, I asked, "Can collaborative groups think?" (Stahl, 2006a, Ch 19) and then at CSCL 2007 looked at the group meaning-making process in some detail (Stahl, 2009, Ch 26).

The VMT research team — along with nine other CSCL labs from around the world—has just published a number of VMT Project case studies of specific group practices (Stahl, 2009). We are now trying to synthesize our findings and - in this paper - to understand how collaborative learning takes place on the basis of computersupported group practices. In particular, four recent case studies show mechanisms of group cognition: resolving differences of perspective or approach (Toledo, Zemel \& Stahl, 2007), engaging in inquiry or questioning (Zhou, Zemel \& Stahl, 2008), creating or maintaining a group problem space (Sarmiento \& Stahl, 2008) and coordinating mathematical problem solving across multiple media for communication or reasoning (Çakir, Zemel \& Stahl, 2009). Here we want to look at the implications of these practices for collaborative learning in our CSCL context. This paper brings together these four illustrative analyses of group practices to show how learning takes place at the small-group level. The question of how this gets individuated-or internalized into 
the minds or practices of the individual students in the groups - is beyond the scope of this paper and of the methodology of the VMT Project.

It is often assumed that case studies do not lead to generalizable findings of theoretical import. Although the following four sections each focus on specific cases of interaction, they should be understood within the contexts of the larger research effort. The four doctoral dissertations (Çakir, 2009; SarmientoKlapper, 2009; Toledo, in preparation; Zhou, 2009) from which these studies are excerpted not only each consider multiple similar cases in detail, but also distill in different ways what has been learned more generally from the VMT Project as a multi-year team-research effort. Our sense of group work informally synthesizes rather diverse data from many virtual math team experiences. The VMT data corpus includes well over a thousand student-hours of chat in 370 session logs, covering a broad array of different experimental contexts. Most of these chats involved K-12 students working on math topics in groups of 3 to 6 . Some involved college students or researchers-occasionally with as many as a dozen participants typing in the same chat room. Students came from around the US, as well as some from Brazil, Singapore and Scotland. Some seemed to be mathematically gifted, but others were probably average and some were at risk. The technology for early VMT sessions consisted of familiar commercial chat systems; by 2005 a system with chat and a shared whiteboard integrated by graphical referencing was used; and in 2006 this was expanded to include a lobby, a tabbed interface and a wiki repository. The math topics evolved from typical algebra and geometry challenge problems from the Math Forum's Problem-of-the-Week (PoW) service to more open-ended topics like the grid world and patterns of sticks and squares.

For a variety of reasons, some of the chat logs are considered better data than others for analyzing the mechanisms of group cognition. In the spring and summer of 2004, an intensive effort was put into coding ten simple chat sessions (PoW-wows). The VMT Spring Fests in 2005 and 2006 brought student groups together for sequences of four hour-long sessions, providing a glimpse into longer-term development of group dynamics and group learning. The four case studies summarized here look at excerpts from teams in the VMT Spring Fest 2005 and 2006 data, as well as going back to an early PoW-wow to look at purely textual interaction. In each case, the specific, highly situated analysis presents a concrete instance of phenomena that are visible-in their rich variety and individuality - throughout the VMT data corpus. These case studies shed light on some of the most theoretically fundamental and elusive themes of CSCL, semiotics, information science and learning science. In particular, each of the four studies addresses a major issue that has been influential in the CSCL research literature. Taken as a whole, they significantly advance our understanding of the nature and mechanisms of group cognition, as will hopefully become clear by the end of this paper.

\section{Case Study \#1: Group-Cognitive Conflict}

The fundamental theories of the learning sciences-going back to the classic texts of both Piaget and Vygotsky - claim that learning is stimulated by an optimal level of differences among conflicting perspectives on a topic. Modern versions of learning theory refer to this claim as "cognitive conflict" - in the socio-cognitive psychological tradition focused on individual cognition (Perret-Clermont \& Schubauer-Leoni, 1981) - and as the "inter-animation of perspectives"-in the socio-cultural dialogical tradition focused on collaborative smallgroup interaction (Wegerif, 2007).

Neo-Piagetian varieties of CSCL, at least, locate the power of collaboration in the attempt to overcome conflicting perspectives, with their attendant psychological tensions. We prefer to deal with the inter-animation of perspectives-the notion that multiple views or approaches can be productive for creative knowledge building in collaborative groups-by looking to see how the alternative perspectives actually interact with each other in group problem-solving efforts. Our analysis illustrates how the eventual resolution of a difference in approach to a problem can drive the group to solve the problem in a way that none of the participants would have individually.

In Group Cognition (Stahl, 2006a, Ch. 21, esp. p. 454f), it was suggested that VMT chats were largely driven forward and sustained by "math proposal adjacency pairs." These are interactions in which one participant makes a proposal bid to the group for the group's work and this is accepted or rejected by another group member on behalf of the group. The studies of resolution of differences look into a more complicated scenario of this interaction: the resolution of differences between two or more math proposals-initiated by different individuals, operating from within contrasting perspectives on the group topic and entering into conflict with each other. The group may take up their conflict and work through it across a longer sequence of postings, rather than just quickly accepting or rejecting a proposal on its own. Such a group activity can drive the work of the group for a significant period of time. The group response to "cognitive conflict" and the subsequent inter-animation of different perspectives can drive learning at both the individual and group level, as it sustains the chat interaction. The result of the resolution of differences can be an expansion of the joint problem space; group participants build a richer shared understanding of the object of their collaborative undertaking. 
While there is widespread agreement on the importance of resolving differences for stimulating learning, there has been little analysis to date of interactional mechanisms by which differences of approach to topics or problems are resolved in small groups. The exploration of such mechanisms requires new qualitative research. It is hard to explore scientifically the resolution of differences in the minds of individuals. However, the resolution of differences within small groups may be observable in traces of their communication and interaction. The VMT Project provides a naturalistic experimental environment that was designed and instrumented to capture the interactions of small groups of students faced with collaborative learning tasks.

Participants in the group problem-solving sessions we have studies engage in a number of activities such as framing the problem or problems, discussing and assessing approaches, executing these approaches and assessing their results as part of performing the activity described as a "problem-solving session." Whether the problem solving is done face-to-face or through computer-mediated communication, as long as there are multiple participants with their respective approaches, procedures and assessment methods, there will need to be some degree of negotiation. Negotiation, defined as "a discussion intended to produce agreement" is a key activity in most group problem solving.

Participants negotiate which approach to use, who is to participate in the unfolding of proffered approaches and in what order competing approaches are to be used. Participants also negotiate how solutions are to be assessed for adequacy and correctness. This interactional process of resolving differences drives the learning activity of the virtual math team by structuring the continuity of the discourse. Participants negotiate when there are competing proposals that appear in their problem-solving interaction. As proposals are advanced, they may be accepted, rejected or ignored. Acceptance is shown in an uptake of the resources offered by the proponent of the proposal. The participants use these resources in similar or compatible ways. Acceptance thus means that the participants build on each other's postings and co-construct their framing of the problem, crafting their solution or assessing the adequacy of their proffered solution. A new posting accepts what was proposed by a previous posting and tries to re-situate it in the new poster's perspective. In the end, the group solves its problem as a result of such back-and-forth motion across differences.

Alternatively, in the face of rejection, participants may adopt other strategies to change the allocation of participation. The spurned proponent may recycle the proposal or post an alternate message, which claims to have some idea that would shed light on the group activity. However, this alternate message would require the other participants to ask the rejected proponent to reveal the idea. If this ploy works, then a counter-proposal may arise and begin another cycle of exchanges. If a proposal is ignored, its proponent may decide to go along with the other proposal, or present a new proposal, or lurk.

These group practices may not appear different from negotiation in a face-to-face setting, since acceptance, rejection or indifference can be communicated through postings as well as through talk. However, in chat acceptance, rejection or indifference may not appear immediately after the proposals to which they would be paired if the interaction were face-to-face. This makes it possible for participants who would otherwise be in an impasse to select parts of a long series of related postings that they can append to their own postings to break an impasse and thereby produce agreement. Thus, in the episode from which the following lines were taken, we find Mario selectively appropriating the postings of Alice and including them in his own presentation, despite his on-going rejection of her approach (Toledo et al., 2007). Similarly, we find Alice using the labels instigated by Mario in making her own contrary claims regarding the reliability of labels. They are tasked with proving why a given geometric situation is impossible, and they propose conflicting approaches:

$\begin{array}{lll}29 & \text { Mario } & \text { You name where the green line meets the base } \\ 30 & \text { Alice } & \text { B } \\ 31 & \text { Alice } & \text { I have an idea that might help us find whats wrong with the pic. } \\ 32 & \text { Mario } & \text { We could use good ol' Pythag thm to see what BV is } \\ 33 & \text { Alice } & \text { Lets not }\end{array}$

Participants recognize agreement when they post tokens of agreement in reaction to other participant's postings. Prior to these displays of agreement, participants show that they are aware that there is some problem, that a solution has to be found, that the solution has to be implemented. The awareness of a problem is expressed in postings that supply additional resources to help frame the problem. For Mario, these additional resources are in the form of labels that eventually frame the problem as a type that can be solved using the Pythagorean theorem. For Alice, labeling is not as consequential. Mario proposes a solution, which is based on the application of the Pythagorean theorem while Alice proposes a different approach to finding a solution. Mario, in proposing the Pythagorean theorem, puts forward an approach that the participants are assumed to be familiar with, while Alice proposes her alternative approach based on details of the given problem description.

We also note that the participants try to negotiate the order in which varying approaches may be applied to the problem at hand. Both Mario and Alice try to get the other participants to apply their approaches first. Both of them work independently and refrain from criticizing each other's approaches until such time as either uses some resource produced by the other to advance their own approach. Thus, Alice uses the labeling 
"BV" that Mario first used to point out how he cannot produce a correct result with his approach. Mario, in return, uses this claim to proceed to a computation of $\mathrm{BV}$, which then produces a result, which is not directly traceable to the use of the Pythagorean theorem but rather to a set of properties associated with equilateral triangles, octagons and hexagons.

If one conceives of the problem solving as the effort of individuals, then one would predict a strong likelihood that this session would have broken down. Two strong willed students brought incompatible approaches to the given task, and each vigorously resisted the approach of the other. However, through the group-interaction processes of negotiation, the differences were resolved in a productive way that led to a solution of the problem and a continuation of the interaction. The resolution of difference did not take place through a vote among preexisting personal opinions, compromise, bargaining or consensus, but through a subtle and selective building of each participant's proposals upon the up-take of the other participant's proposals. A shared framing of the problem - or a joint problem space-was co-constructed through the inter-animation of alternative perspectives on the problem. Through fine-grained analysis of the chat log, it was possible to characterize various interactional methods that were employed by the group to achieve a productive interanimation.

The excerpt that was analyzed can be seen to have been driven forward by the interactive moves between participants, motivated by their different perspectives. From a methodological viewpoint, it is important to note that the driving force is not the individuals as agents, but the tension between them. The math solution does not arise directly from the mental representations of the individual students, but from the group effort to respond to the conflicting differences and from the interplay between the participants. Of course, the brains of each student were necessary to interpret the group meanings created in the interaction and to articulate the utterances that were posted in the chat in response to the on-going discourse, but the problem framing, the group problem space, the solution path, the meaning making all took place at the group level in the visible, persistent chat.

What can be said about learning in this case study? If we talk about the group learning-having followed a path to that solution and having arrived at an understanding of the solution of the problem-then we can say that the group learning was driven by the process of interactively resolving the differences of proposed approaches. If, further, we assume that the individual students learned something from the experience, we can say they did so by "individuating" the group lesson, making it their own and integrating it into their personal understanding, where it can serve as a set of resources for future mathematical discourses (including internal discourses of thought). Because the effort to resolve differences in the chat discourse kept both Alice and Mario focused on the proposals of the other, it is likely that they will each internalize something of their opponent's perspective. In this sense, their individual learning will be driven by the confrontation with a perspective that conflicted with their own. Experiences like these could lead to their ability to learn on their own by reading and even by thinking about perspectives that conflict with their own initial ideas. Thus, analysis of this case study seems to provide insight into grand theories of individual and collaborative learning through cognitive conflict and inter-animation of perspectives as driven by the resolution of differences.

\section{Case Study \#2: Questioning to Learn}

The study of practices of group questioning investigates another driving force of collaboration. Rather than seeing a question posed in a chat as an outward expression of an individual's mental idea or of an individual's request for information, we look at the methods of formulating and taking up a bid at questioning to see how the meaning and function of the questioning are negotiated interactively. Questioning is seen to be a potentially complex group process, incorporating a wide variety of interactional methods. A question can be part of a math proposal adjacency pair, putting forward a tentative proposal or reacting to a proposal bid. Questioning within a group can extend across a much longer sequence of adjacency pairs, advancing (or not) the problem-solving trajectory of the group. This analysis of questioning as an interactional achievement of a group - as opposed to a query in an individual mind-signals an innovative interactional approach to information science, with its conceptualizations of knowledge and information seeking that often underlie CSCL theories.

In an online collaborative context like VMT chats, questions are often not simple, well-defined queries for pre-existing information, but should be understood as situated moves within the group dynamic of the problem-solving effort. The object of the questioning is itself an emergent property of the interaction, through which the meaning is successively interpreted, refined and converged upon by the details of how the question is built, read and responded to. Questioning can play an integral role in the social relations among the participants, either positioning individuals as more or less competent or else maintaining peer standings. Question/response interactions are key to pursuing group problem-solving strategies, building a joint problem space and sustaining the team discourse.

We start by asking how it is possible to sustain a productive peer relationship in an online group when the raising of questions often reveals and makes relevant differences among actors in expertise, talent, ability, knowledge or understanding. Pursuing this line of inquiry allows us to look into the mechanisms underlying 
peer-group interaction. When there are differences in competence, actors need to work out among themselves the social order and the organization of their interaction. We look at how differences are attended to by participants in a collaborative peer group as part of the mechanism by which a group of students collaborate and manage the organization of their participation in ongoing chat interaction around problem solving. In particular, we examine the ways members of a small group (a) introduce differences in situated competencies as interactionally relevant, (b) organize their interaction to attend to these differences and (c) effect repairs where possible or find ways to proceed where repair is ineffective.

There are many ways that differences in competency can be introduced as interactionally relevant. Posing a question is often one way of accomplishing this. For example, an actor can ask a question about what is going on, or indicate there is a problem of understanding, or the actor can show the need for assistance by taking a particular kind of "next step" in a sequentially unfolding set of actions, for instance. When a questioner asks certain kinds of questions, she constitutes and makes relevant differences in expertise, knowledge, etc. as a matter for the recipients to attend to. Thus, not only is the questioner asking a recipient about the matter at hand, she is also instantiating their relationship in terms of the organization of their participation in the interaction (e.g., as questioner and answerer). In examining our data of students' interaction in VMT chats, we have noticed that question-response pairs are frequently invoked for attending to differences in local expertise and competency. For instance, asking a question may imply that the addressee(s) are likely to be able to provide some information that the questioner does not know.

When actors put forward certain questions that do not address explicitly their standing as participants in the interaction, matters of difference in knowledge, understanding, expertise, etc., can be addressed in ways that preserve a peer relationship between questioner and respondent. When actors make the organization of participation explicit in the question-response construction as a matter to be addressed, then the nature of the relationships among interactants becomes a matter of concern that needs to be addressed. Issues of differences in knowledge, understanding or expertise are then made relevant in terms of the way those relationships are worked out. In the following excerpt (Zhou et al., 2008), Nish positions himself as potentially "stupid" sounding; this lessens the possibility that respondents will position him as being less competent and will simply provide the requested explanation. The respondents, 137 and Jason, respond with relevant resources, without putting themselves in a teacher role. However, in line 180 Jason makes explicit the difference in math competency level between Nish and the rest of the group, effectively excluding Nish from full participation in the group work.

$\begin{array}{lll}175 & \text { Nish } & \text { hope this doesnt sound too stupid, but wuts a summation } \\ 177 & 137 & \text { The sum of all terms from a to } b \\ 178 & \text { Jason } & \text { http://en.wikipedia.org/wiki/Sigma_notation } \\ 180 & \text { Jason } & \text { don't worry Nish, you'll learn all about it next year }\end{array}$

In analogy to our analysis of a "failed proposal" in our discussion of math proposal adjacency pairs (Stahl, 2006a, Ch. 21, esp. p. 454f), we contrasted a "breakdown" example of a question-response interaction to a successful case in an attempt to specify the characteristics of a "successful question." The analysis suggests the following characteristics, some of which bear resemblance to those for successful proposals:

(a) A clear question structure that elicits a response. Making a report of one's math competency (beginning of line 175) may indicate some problem of understanding, but not present a question of its own. It does not elicit a response from the group. A question on a math topic with a clear structure is more likely to elicit a response without interactional trouble.

(b) Information on what is known by the questioner. A question such as "what's a summation?" may be ambiguous as to what it is really asking for, as there are multiple possible readings of it. Providing information on what the questioner already knows can help rule out some possible readings of the question.

(c) Right timing and interactional context within the sequence of interaction. Posing a question irrelevant to the ongoing discussion takes the risk of interrupting the group and deviating from the topic; careful work is needed to build the context for the question; ignoring this risks failure.

(d) Engagement in the group process. Indication of being engaged in the group process is also helpful in that it contributes to enacting and maintaining the peer relationship. Failing to engage in the group process like Nish does during the response construction can be destructive to the peer relationship.

Question-response interactions are key to pursuing group problem-solving strategies, building a group problem space and sustaining the team discourse. Participants do not just pose questions as information-seeking or helpseeking moves by individuals. Question-response pairs also function at the small-group level as mechanisms for managing peer relationships and organizing participation. They can function to include — or exclude - a group member. They can play an integral role in the social relations among the participants, positioning individuals as more or less competent and maintaining or adjusting peer standings. 


\section{Case Study \#3: Evolving the Joint Problem Space}

In order to engage in shared work as a group, there must be a task to work on together-what activity theory refers to as the "object" of the group activity. This must be more than simply a statement of a problem that was given to the group, but needs to be worked out as a "problem space" to which the group can orient itself in an on-going and practical way. We looked at how a group establishes and maintains its "joint problem space" (Sarmiento \& Stahl, 2008). Our study grew out of an attempt to understand how groups maintain their continuity of interaction across discontinuities. It extended our understanding of how a joint problem space is maintained by stressing the sequential and temporal aspects of "bridging" methods that are typically employed by virtual math teams to overcome discontinuities that threaten to disrupt their effort. We now see the joint problem space as integrating: (a) social aspects (which transform participants into "members" of the interactional group), (b) domain content concerns (such as the group's characterization of their problem to be solved) and (c) temporal relations (the past, present and future as they are constituted in the unfolding sequentiality of the group interaction). This joint problem space structures the work and discourse of the group, providing a shared understanding of the references and concerns that are expressed in utterances and behaviors of the individual group members. This analysis replaces the easily misunderstood metaphor of common ground with a richer construct.

Theories of collaborative learning have identified the central role of the joint problem space (JPS) in coordinating work and establishing intersubjective understanding (Teasley \& Roschelle, 1993). The concept of problem space had its inception within the information-processing perspective as a characterization of individual problem-solving activity. It was then reformulated and extended within the learning sciences to include the social and domain dimensions. Based on a detailed analysis of sustained online collaborative problem-solving activity by a small group of students over multiple sessions, we propose that the theory of the joint problem space should now be further expanded. In addition to the dimensions of social relations and domain content, which are increasingly recognized in the learning sciences, we argue for the salience of the temporal dimension. Our analysis shows that the joint problem space is co-constructed at the group unit of analysis through the temporal and sequential orientation to inter-subjective meaning making.

The JPS can now be seen as a socio-temporal-semantic field, co-constructed through interactions such as collective remembering and providing the basis for shared understanding of meaning. Processes of group cognition both sustain and are sustained by the JPS. The JPS is seen as an interactional phenomenon at the small-group unit of analysis, rather than as a convergence of mental representations of individuals as is often understood within theories of cognitive change and common ground. That is, the JPS is established and maintained through the sequential relationship of interactions among group participants as they build upon past actions, current situations and future opportunities of their group activity. Individual mental representations are possible spin-offs of the JPS, rather than causes of it.

All of these resources - the knowledge artifacts used and referenced, the sequential organization of cases and the temporal markers of prior activity-are organized in different ways with relation to the participants in a temporal or sequential space. The concept of "deictic field" developed by Hanks (2005) seems especially useful to define the relationship between this new "space" and Barron's domain content and social relational spaces (Barron, 2003). Hanks describes the deictic field as composed first by "the positions of communicative agents relative to the participant frameworks they occupy," for example, who occupies the positions of speaker and addressee as well as other relevant positions. Second, the deictic field integrates "the positions occupied by objects of reference," and finally "the multiple dimensions whereby the former have access to the latter" (p. 193). From this perspective, participants in the following excerpt constitute, through interaction, the relevant relative dimensions whereby they are to manage the positioning of agents and relevant objects of reference. They collectively co-construct a field of spatio-temporal indexicality incorporating bridging across sessions to locate activities, events and resources.

$\begin{array}{lll}144 & \text { mathis } & \text { letz start working on number } 8 \\ 145 & \text { bob1 } & \text { we already did that yesterday } \\ 146 & \text { qw } & \text { we did? } \\ 147 & \text { mathis } & \text { but we did it so that there was only right and down } \\ 148 & \text { bob1 } & \text { i mean tuesday } \\ 149 & \text { mathis } & \text { i guess we will do it with left and up? } \\ 150 & \text { qw } & \text { It would be almost the same. }\end{array}$

In the interaction excerpted here, the three dimensions are intimately intertwined or unified. Participation is managed so that people who were or were not present in the previous session could nevertheless be included in remembering the knowledge constructed then. The knowledge artifacts (paths, formulae, procedures for exploring patterns) of the past are situated in the present work. The temporal discontinuity between sessions is bridged and the sequentiality of the group work is organized within the newly elaborated deictic field that the group incorporated in their joint problem space. 
In our analysis of interactions we have observed that the content and relational dimensions are, in fact, relevant to collaborative problem-solving teams. Moreover, in expanding the range of phenomena analyzed to include longitudinal interactions across discontinuities, we have also uncovered time and the sequential unfolding of interaction as a third relevant and important dimension of activity. The interactional field is constituted by the participants to include problem-related objects and communicative agents associated with a prior interaction, and in doing so they position themselves and those resources within specific participation frameworks. The content objects (e.g., knowledge artifacts) and the relations among people (e.g., social positioning) are located within a temporal field, which provides a context for situating past, present and future events, for pointing to the events as temporally structured and for ordering utterances in their sequential relationships. Our central claim is that this temporal/sequential dimension is as essential to understanding collaborative interactions as are the content and relational dimensions.

The theory of group cognition takes as one of its central principles the dialectical relationship between social interaction and the construction of meaning. Meaning is not viewed as pre-existing in the minds of individuals, but as something that is constituted in the discourse within the group (Stahl, 2006a, Ch. 16). Nor is the group viewed as pre-existing as a set of people, but as a functional unit that constitutes itself in the interaction of its members when they position themselves within their group activity. From this perspective, the social organization of action and the knowledge embedded in such action are emergent properties of momentby-moment interactions among actors, and between actors and the objects and the activity systems in which they participate collectively. The content space and the relational space, in Barron's terms, are mutually constitutive from this perspective.

Group cognition theory offers a candidate description for how the dynamic process of building knowledge might intertwine the content and relational spaces: "Small groups are the engines of knowledge building. The knowing that groups build up in manifold forms is what becomes internalized by their members as individual learning and externalized in their communities as certifiable knowledge" (Stahl, 2006a, p. 16). Thus, small group interaction can play a pivotal mediating role in the interplay between individual cognition (and the relations among the individuals) and communities of practice (and the knowledge objects that they share). Time as the sequential organization of activity seems to be a resource and an aspect of interaction that plays a significant role in how communities, groups and individuals achieve knowledge through small-group interaction. We have caught a glimpse or two of how temporality is marked and sequentiality is established within the discourse of small groups in VMT.

In our analysis of how small groups "sustain" their group cognition while engaged in brief episodes of online mathematical problem solving, we alluded to two ways in which time might be an important element of individual episodes of problem-solving activity. On the one hand, the collaborative activity involved in solving a problem can be "spread across" hundreds of micro-level interactions. On the other hand, individuals might internalize or individualize the meaning co-constructed through interactions and "sustain" the group cognition by engaging in later individual or group work. In either case, groups are described as sustaining their social and intellectual work by "building longer sequences of math proposals, other adjacency pairs and a variety of interaction methods" (Stahl, 2006b, p. 85).

Our analysis of interactions that bridge gaps across sessions confirms and extends these findings by suggesting that in longitudinal interactions, temporal and sequential resources are central to constituting activity as continuous by constructing and maintaining a group problem space. Interaction is taken here in the full sense that ethnomethodologists give it, as the "ongoing, contingent co-production of a shared social/material world," which, as Suchman argues "cannot be stipulated in advance, but requires an autobiography, a presence and a projected future" (Suchman, 2003). We have just began the work of describing in detail the interactional group practices that allow teams to construct and manage this expanded problem "field" by interweaving content, relational and temporal aspects of interaction.

\section{Case Study \#4: Coordinating Visual, Narrative and Symbolic Reasoning}

We now consider how work in the group problem space is conducted when the online environment combines textual postings and graphical drawing media, as in a VMT chat room with shared whiteboard. By looking closely at the practices a student group uses to coordinate chat postings with carefully choreographed inscriptions on the shared whiteboard, we see how deep understanding of math can be effectively promoted through the organization of visual, narrative and symbolic reasoning within group interaction. Although drawings, text and mathematical symbols build knowledge and convey meaning through very different semiotic systems, in VMT sessions they are tightly coordinated and mutually informing. Students new to the environment spontaneously develop and share methods of connecting and coordinating work in these media.

Mathematical insight is often first grounded in visual reasoning with concrete instances, where relationships can be seen and understood concretely. These insights can then be pointed out to others through narratives, which instruct them how to see in the group's shared way. In mathematics, symbolic expressions are effectively employed to articulate, formalize and generalize understandings of relationships, providing means 
for symbolic manipulations that lead to further conclusions and to different forms of comprehension. The math artifacts that emerge from group work that coordinates visual, narrative and symbolic reasoning are not simple objects, but concepts that can only be understood through the coordination of their multiple realizations in these different types of media. The coordination of group work in the three realms supports deep mathematical understanding (as opposed to rote learning) of individuals by fostering understanding of the multiple realizations of math artifacts. It also enriches the joint problem space of the group's effort by interconnecting the semantic relationships of the three realms within a shared network of meaning.

We recently investigated how a group of three upper-middle-school students put the features of an online environment with dual interaction spaces into use as they collaboratively worked on a math problem they themselves came up with (Çakir et al., 2009). Our analysis revealed several important insights regarding the affordances of systems with dual interaction spaces. First, we observed that the whiteboard can make visible to everyone the animated evolution of a geometric construction, displaying the visual reasoning process manifested in drawing actions. Second, whiteboard and chat contents differ in terms of mutability of their contents, due to the object-oriented design of the whiteboard, which allows modification and annotation of past contributions. Third, the media differ in terms of the persistence of their contents: whiteboard objects remain in the shared visual field until they are removed, whereas chat content gradually scrolls off as new postings are produced. Although contents of both spaces are persistently available for reference, due to linear progression of the chat window, chat postings are likely to refer to visually (and hence temporally) proximal chat messages and to graphical whiteboard objects. Finally, the whiteboard objects index a horizon of past and future activities as they serve as an interactional resource through the course of related episodes of chat discussion.

Our analysis of this team's joint work also revealed methods for the organization of collaborative work, through which group members co-construct mathematical meaning sedimented in semiotic objects distributed across the dual interaction spaces of the VMT environment. We observed that bringing relevant math artifacts referenced by indexical terms such as "hexagonal array" to other members' attention often requires a coordinated sequence of actions across the two interaction spaces. Participants use explicit and verbal references to guide each other about how a new contribution should be read in relation to prior contents. Indexical terms stated in chat referring to the visible production of shared objects are instrumental in the reification of those terms as meaningful mathematical objects for the participants. Verbal references to co-constructed graphical objects are often used as a resource to index complicated mathematical concepts in the process of coconstructing new concepts. Finally, different representational affordances of the dual interaction spaces allow groups to develop multiple realizations of the math artifacts to which they are oriented. Shared graphical inscriptions and chat postings are used together as semiotic resources in mutually elaborating ways. Methods of coordinating group interaction across the media spaces also interrelate the mathematical significances of the multiple realizations.

Overall, we observed that actions performed in both the chat and whiteboard interaction spaces constitute an evolving historical context for the joint work of the group. What gets done now informs the relevant actions to be performed next, and what was done previously can be reproduced/modified depending on the circumstances of the ongoing activity. As the interaction unfolds sequentially, the sense of previously posted whiteboard objects and chat statements may become evident and/or modified, as in this brief excerpt:

12

13

14

15

16

17

18

19

20
137

Qwertyuiop
137
Qwertyuiop
Jason
Jason
Qwertyuiop
Jason
Jason

\author{
So do you want to first calculate the number of triangles in a \\ hexagonal array?

Here the VMT environment's graphical referencing tool is used to coordinate chat postings with previous chat postings as well as with objects on the whiteboard. Through the sequential coordination of chat postings and whiteboard inscriptions, the group successfully solved their self-defined mathematical challenge, to find a formula for the number of small triangles in a hexagonal array of any given side-length. Their interaction was guided by a sequence of proposals and responses carried out textually in the chat medium. However, the sense of the terms and relationships narrated in the chat were largely instantiated, shared and investigated through observation of visible features of graphical inscriptions in the whiteboard medium. The mathematical object that was visually co-constructed in the whiteboard was named and described in words within the chat. Finally, a symbolic expression was developed by the group, grounded in the graphic that evolved in the 
whiteboard and discussed in the terminology that emerged in the chat. The symbolic mathematical result was then posted to the wiki, a third medium within the VMT environment. The wiki is intended for sharing group findings with other groups as part of a permanent archive of community knowledge building by virtual math teams.

Our case study demonstrates that it is possible to analyze how math problem solving - and presumably other learning achievements - can be carried out by small groups of students. The students can define and refine their own problems to pursue; they can invent their own methods of working; they can use unrestricted vocabulary; they can coordinate work in multiple media, taking advantage of different affordances. Careful attention to the sequentiality of references and responses is necessary to reveal how the group coordinated its work and how that work was driven by the reactions of the group members' interactions with each other. Only by focusing on the sequentiality of the interactions can one see how the visual, narrative and symbolic build on each other as well as how the actions of the individual students respond to each other to co-construct math objects, personal understanding, group agreement and mathematical results that cannot be attributed to any one individual, but which emerge from the interaction as complexly sequenced. This analysis illustrates a promising approach for CSCL research to investigate aspects of group cognition that are beyond the reach of quantitative methods that ignore the full sequentiality of their data.

In our case study, we have seen the establishment of an indexical ground of deictic references coconstructed by the group members as an underlying support for the creation and maintenance of their joint problem space. We have seen that nexus of references created interactionally as group members propose, question, repair, respond, illustrate, make visible, supply symbols, name, etc. In the VMT dual-media environment, the differential persistence, visibility and mutability of the media is consequential for the interaction. Group members develop methods of coordinating chat and drawing activities to combine visual and conceptual reasoning by the group and to co-construct and maintain an evolving shared indexical ground of their discourse.

During the 18 minute excerpt analyzed in this case study, three students construct a diagram of lines, triangles and hexagons, propose a math pattern problem, analyze the structure of their diagram and derive an algebraic formula to solve their problem. They do this by coordinating their whiteboard and chat activities in a synchronous online environment. Their accomplishment is precisely the kind of educational math experience recommended by mathematicians (Livingston, 2006; Lockhart, 2008; Moss \& Beatty, 2006). It was not a mental achievement of an individual, but a group accomplishment carried out in computer-supported discourse. By analyzing the sequentiality and indexicality of their interactions we explicated several mechanisms of this group cognition by which the students coordinated the meaning of their discourse and maintained adequate reciprocity of understanding.

The coordination of visual and semiotic realizations of the mathematical objects that the students coconstruct provides a grounding of the algebraic formulas the students jointly derive in the line drawings that they inspect visually together. As the students individualize this experience of group cognition, they can develop the deep understanding of mathematical phenomena that comes from seeing the connections among multiple realizations (Sfard, 2008). Our case study does not by any means predict that all students can accomplish similar results under specific conditions, but merely demonstrates that this is possible within a synchronous CSCL setting and that a fine-grained sequential analysis of interaction can study how the group accomplished it.

\section{Group Cognition and Learning}

As a research field, CSCL has been deeply influenced by the theories of Vygotsky (1930/1978). In particular, one can say that CSCL is inspired by his visionary insight that learning takes place originally inter-subjectively (in small groups), and may then be internalized as intra-subjective (individual) learning. To this view, CSCL adds the hope that networked computer technology can bring learners together in new ways to take advantage of the power of collaborative learning. In this paper, we have tried to indicate a way of analyzing group learning that was not available to Vygotsky and that has been too little pursued within CSCL to date. By observing the group practices through which small groups of learners accomplish problem solving and other tasks, we can begin to determine the mechanisms that make knowledge building possible at the small-group level. We can observe group practices with the requisite detail by recording interactions that take place in CSCL settings, where the complete context of interaction can be captured, logged and replayed for analysis. Then we can describe the kinds of interactions that take place in group-cognitive conflict, in group inquiry, in maintaining a group problem space or in coordinating group reasoning across multiple media. These group practices set the stage for individual learning by allowing groups to reach achievements that the group's members can take away as skills, resources or methods for their own learning. As Vygotsky noted, the mediations involved in internalization are complex - and we would add that they are hard to observe. However, to understand individual learning as a cultural and developmental process, it seems necessary — and quite possible - first to understand the practices of group cognition that underlie it. We may then find that the traditional conceptualizations of individual learning must be reworked on the model of the small-group practices. 


\section{References}

Barron, B. (2003). When smart groups fail. The Journal of the Learning Sciences, 12(3), 307-359.

Çakir, M. P. (2009). How online small groups co-construct mathematical artifacts to do collaborative problem solving. Unpublished Dissertation, Ph.D., College of Information Science and Technology, Drexel University, Philadelphia, PA, USA.

Çakir, M. P., Zemel, A., \& Stahl, G. (2009). The joint organization of interaction within a multimodal CSCL medium. International Journal of Computer-Supported Collaborative Learning (ijCSCL), 4(2).

Cohen, E. G., Lotan, R. A., Abram, P. L., Scarloss, B. A., \& Schultz, S. E. (2002). Can groups learn? Teachers College Record, 104(6), 1045-1068.

Lave, J., \& Wenger, E. (1991). Situated learning: Legitimate peripheral participation. Cambridge, UK: Cambridge University Press.

Livingston, E. (2006). Ethnomethodological studies of mediated interaction and mundane expertise. The Sociological Review, 54(3).

Lockhart, P. (2008). Lockhart's lament. MAA Online, 2008(March). Retrieved from http://www.maa.org/devlin/devlin 03 08.html.

Moss, J., \& Beatty, R. (2006). Knowledge building in mathematics: Supporting collaborative learning in pattern problems. International Journal of Computer-Supported Collaborative Learning, 1(4), 441-465. Retrieved from http://dx.doi.org/10.1007/s11412-006-9003-z.

Perret-Clermont, A.-N., \& Schubauer-Leoni, M.-L. (1981). Conflict and cooperation as opportunities for learning. In W. P. Robinson (Ed.), Communication in development (pp. 203-234). New York, NY: Academic Press.

Sarmiento, J., \& Stahl, G. (2008). Extending the joint problem space: Time and sequence as essential features of knowledge building. Paper presented at the International Conference of the Learning Sciences (ICLS 2008), Utrecht, Netherlands. Retrieved from http://GerryStahl.net/pub/icls2008johann.pdf.

Sarmiento-Klapper, J. W. (2009). Bridging mechanisms in team-based online problem solving: Continuity in building collaborative knowledge. Unpublished Dissertation, Ph.D., College of Information Science and Technology, Drexel University, Philadelphia, PA, USA.

Schwartz, D. (1995). The emergence of abstract representations in dyad problem solving. Journal of the Learning Sciences, 4(3), 321-354.

Sfard, A. (2008). Thinking as communicating: Human development, the growth of discourses and mathematizing. Cambridge, UK: Cambridge University Press.

Stahl, G. (2006a). Group cognition: Computer support for building collaborative knowledge. Cambridge, MA: MIT Press. Retrieved from http://GerryStahl.net/mit/.

Stahl, G. (2006b). Sustaining group cognition in a math chat environment. Research and Practice in Technology Enhanced Learning (RPTEL), 1(2), 85-113. Retrieved from http://GerryStahl.net/pub/rptel.pdf.

Stahl, G. (Ed.). (2009). Studying virtual math teams. New York, NY: Springer. Computer-supported collaborative learning book series, vol 11 Retrieved from http://GerryStahl.net/vmt/book.

Teasley, S. D., \& Roschelle, J. (1993). Constructing a joint problem space: The computer as a tool for sharing knowledge. In S. P. Lajoie \& S. J. Derry (Eds.), Computers as cognitive tools (pp. 229-258). Mahwah, NJ: Lawrence Erlbaum Associates, Inc.

Toledo, R. (in preparation). How the resolution of differences drives problem solving in virtual math teams. Unpublished Dissertation, Ph.D., College of Information Science and Technology, Drexel University, Philadelphia, PA, USA.

Toledo, R. P. S., Zemel, A., \& Stahl, G. (2007). Resolving differences: Twists and turns in a synchronous online collaborative mathematics problem-solving session. Paper presented at the international conference on Computer-Supported Collaborative Learning (CSCL '07), New Brunswick, NJ. Retrieved from http://GerryStahl.net/vmtwiki/ramon.pdf.

Vygotsky, L. (1930/1978). Mind in society. Cambridge, MA: Harvard University Press.

Wegerif, R. (2007). Dialogic, education and technology: Expanding the space of learning. New York, NY: Kluwer-Springer.

Zhou, N. (2009). Investigating information practices of collaborative online small groups engaged in problem solving. Unpublished Dissertation, Ph.D., College of Information Science and Technology, Drexel University, Philadelphia, PA, USA.

Zhou, N., Zemel, A., \& Stahl, G. (2008). Questioning and responding in online small groups engaged in collaborative math problem solving. Paper presented at the International Conference of the Learning Sciences (ICLS 2008), Utrecht, Netherlands. Retrieved from http://GerryStahl.net/pub/icls2008nan.pdf. 